\title{
EFICACIA DE DOS FORMULACIONES DE OXFENDAZOL PRODUCIDAS LOCALMENTE PARA EL TRATAMIENTO DE LA CISTICERCOSIS EN CERDOS INFECTADOS NATURALMENTE
}

\author{
Gianfranco Arroyo(1),a,b, Javier A. Bustos (1),c,d, Juan F. Calcina(102,a, \\ Linda Gallegos $\mathbb{1}^{2, a}$, Ana Vargas-Calla ${ }^{2, a, b}$, Luis A. Gomez-Puerta 2a,a,

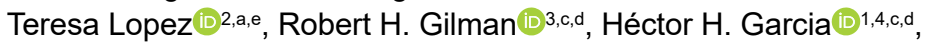 \\ Armando E. Gonzalez ${ }^{2, a, e}$, Grupo de Trabajo en Cisticercosis en Perú. \\ 1 Centro de Salud Global y Departamento de Microbiología, Universidad Peruana Cayetano Heredia, Lima, Perú. \\ 2 Facultad de Medicina Veterinaria, Universidad Nacional Mayor de San Marcos, Lima, Perú. \\ 3 Department of International Health, Bloomberg School of Public Health, Johns Hopkins University, Baltimore, Maryland, \\ Estados Unidos. \\ ${ }^{4}$ Unidad de Cisticercosis, Instituto Nacional de Ciencias Neurológicas, Lima, Perú. \\ ${ }^{a}$ Médico veterinario; ${ }^{\mathrm{b}}$ magíster en Salud Animal; ${ }^{\mathrm{c}}$ médico cirujano, ${ }^{\mathrm{d}}$ doctor en Salud Pública, ${ }^{\mathrm{e}}$ doctor en Medicina \\ Veterinaria.
}

\section{RESUMEN}

Se evaluó la eficacia de dos formulaciones de oxfendazol (OFZ) contra cisticercosis producidas localmente, al 22,5\% y $10 \%$ en comparación con una formulación comercial (Synanthic 9,06\%) en 22 cerdos naturalmente infectados, que recibieron una dosis oral de $30 \mathrm{mg} / \mathrm{kg}$. Los cerdos fueron sacrificados a las ocho semanas postratamiento para evaluar quistes en en sus carcasas, y se determinó la eficacia cisticida a través de la proporción de quistes degenerados sobre el total. Solo se encontraron quistes degenerados en la musculatura, corazón y lengua de los cerdos tratados con OFZ en todos los grupos, lo cual muestra una eficacia del $100 \%$. En los cerebros se encontraron quistes viables y degenerados, con una eficacia menor en todos los grupos (65\% [OFZ comercial], 47\% [OFZ local 22,5\%] y 31\% [OFZ local 10\%], $\mathrm{p}=0,355$. Las formulaciones de OFZ producidas localmente fueron igual de efectivas que la formulación comercial y pueden proporcionar una alternativa para el tratamiento de la cisticercosis porcina.

Palabras claves: Cisticercosis; Dosificación; Quistes; Porcinos; Taenia solium; Peru (fuente: DeCS BIREME).

\section{EFFICACY OF TWO LOCALLY PRODUCED OXFENDAZOLE FORMULATIONS FOR THE TREATMENT OF CYSTICERCOSIS IN NATURALLY INFECTED PIGS}

Citar como: Arroyo G, Bustos JA, Calcina JF, Gallegos L, Vargas-Calla A, Gomez-Puerta L, et al. Eficacia de dos formulaciones de oxfendazol producidas localmente para el tratamiento de la cisticercosis en cerdos infectados naturalmente. Rev Peru Med Exp Salud Publica. 2021;38(2):296301. doi: https://doi.org/10.17843/ rpmesp.2021.382.6539.

\section{Correspondencia:}

Ana Vargas-Calla; Facultad de Medicina Veterinaria, Universidad Nacional Mayor de San Marcos, Lima, Perú; anavargascalla@outlook.com

Recibido: 05/10/2020 Aprobado: 23/06/2021 En Línea: 07/07/2021

\begin{abstract}
The efficacy of two locally produced oxfendazole (OFZ) formulations against cysticercosis at $22,5 \%$ and $10 \%$, versus a commercial formulation (Synanthic 9,06\%) was evaluated in twenty-two naturally infected pigs that received a single oral dose of $30 \mathrm{mg} / \mathrm{kg}$. Pigs were sacrificed at eight weeks post-treatment to evaluate the cysts found in their carcasses, and to determine the cysticidal efficacy, which was defined as the proportion of degenerated cysts over total cysts. Only degenerated cysts were found in muscle, heart, and tongue of pigs treated with OFZ in all groups, which shows an efficacy of $100 \%$. Viable and degenerated cysts were found in brains, being the efficacy lower in all groups (65\% [commercial OFZ], 47\% [local OFZ 22.5\%] and 31\% [local OFZ $10 \%], p=0.355$ ). Locally produced OFZ formulations were similarly effective to the commercial formulation and may provide a practical alternative for the treatment of porcine cysticercosis.
\end{abstract}

Keywords: Cysticercosis, Dosage, Cysts, Porcine, Taenia solium, Peru (source: MeSH NLM).

\section{INTRODUCCIÓN}

Taenia solium es un céstodo zoonótico y endémico en países en vías de desarrollo. El ciclo de vida de T. solium involucra a humanos y cerdos como hospedadores definitivos e interme- 
dios respectivamente. En los humanos el estadio adulto de la tenia se desarrolla en el intestino delgado cuando ingieren carne de cerdo cruda o mal cocida, infectada con quistes. Por otro lado, en los cerdos se desarrolla el estadio larvario (cisticerco) al ingerir heces humanas que contengan huevos de $T$. solium ${ }^{(1)}$. Los humanos también pueden desarrollar cisticercosis al ingerir accidentalmente estos huevos ${ }^{(1)}$. La neurocisticercosis (NCC), la infección del sistema nervioso central por la forma larvaria (cisticerco), es la principal causa de epilepsia adquirida en humanos en todo el mundo ${ }^{(2)}$ y ocasiona un impacto económico negativo en los pacientes debido a los costos de hospitalización y pérdidas de productividad $^{(3)}$.

Las intervenciones combinadas dirigidas tanto a poblaciones humana y porcina tienen el potencial de interrumpir el ciclo de transmisión de T. solium. La quimioterapia con oxfendazol en cerdos (OFZ) ha demostrado ser altamente eficaz contra la cisticercosis porcina ${ }^{(4)}$. El OFZ (5 - [(fenil-sulfinilo) -1H-bencimidazol-2-il] éster metílico del ácido carbámico) es un benzimidazol de amplio espectro contra nematodos y trematodos en diversas especies animales a dosis que oscilan entre 2 y $10 \mathrm{mg} / \mathrm{kg}^{(5)}$. Estudios controlados demostraron la eficacia cisticida del OFZ contra la cisticercosis porcina a una dosis oral única de $30 \mathrm{mg} / \mathrm{kg}{ }^{(6)}$ y posteriores ensayos de campo con OFZ, solo o combinado con la vacunación contra cisticercosis porcina, han corroborado su eficacia ${ }^{(4,7,8)}$.

La farmacocinética del OFZ en cerdos que recibieron una dosis oral única de $30 \mathrm{mg} / \mathrm{kg}$ de una formulación comercial (Synanthic 9,06\%, Pfizer, México) y de una formulación experimental local al 22,5\% mostraron concentraciones plasmáticas muy altas dos horas después del tratamiento ${ }^{(9)}$. Tanto la concentración máxima como el área bajo la curva de la concentración-tiempo fueron aproximadamente un $30 \%$ más altas para la formulación comercial; sin embargo, no se indicó si estas diferencias pueden explicar su eficacia cisticida. Actualmente, el OFZ tiene licencia para su uso contra la cisticercosis porcina en África ${ }^{(8)}$. En Perú se comercializa el OFZ a concentraciones de $10 \%$ o menos. Estas formulaciones poco concentradas requieren un volumen grande de medicación por cerdo y tienen el riesgo de aspiración y muerte del animal durante el tratamiento. Las formulaciones más concentradas de OFZ serían más fáciles de administrar en programas de quimioterapia porcina masiva, ya que disminuyen los riesgos de aspiración, pero no están disponibles en el mercado. Si las formulaciones locales más concentradas de OFZ demuestran una eficacia similar, podrían proporcionar una alternativa práctica. Este experimento controlado comparó la eficacia de dos formulaciones de OFZ producidas localmente $(22,5 \%$ y $10 \%)$ y una formulación comercial contra cisticercosis porcina en cerdos infectados naturalmente.

\section{MENSAJES CLAVE}

Motivación para realizar el estudio: El oxfendazol (OFZ) es un antiparasitario efectivo contra la cisticercosis porcina y una herramienta valiosa para su control, aunque el volumen requerido por animal a la concentración usual de $9 \%$ lo hace impráctico para su administración en masa.

Principales hallazgos: Las formulaciones de OFZ producidas localmente fueron igualmente efectivas que la formulación comercial.

Implicancias: Las formulaciones locales de OFZ pueden proporcionar una alternativa para el tratamiento de la cisticercosis porcina en áreas rurales.

\section{EL ESTUDIO}

El presente estudio experimental se realizó en las instalaciones de la Facultad de Medicina Veterinaria de la Universidad Nacional Mayor de San Marcos (FMV-UNMSM) en Lima, Perú. Veintidós cerdos, mayores de 12 meses infectados con cisticercosis y diagnosticados mediante examen de lengua ${ }^{(10)}$, fueron comprados en Huancayo, un área endémica de cisticercosis de la sierra peruana ${ }^{(11)}$. Los cerdos fueron trasladados hacia las instalaciones experimentales, vacunados contra el virus del cólera porcino y aretados con números de identificación dos semanas antes del inicio del estudio. Los cerdos fueron asignados aleatoriamente (en estratos por peso) para recibir una dosis oral única de $30 \mathrm{mg} / \mathrm{kg}$ del OFZ comercial (Synanthic 9,06\%, Pfizer, México ${ }^{\circledR}[\mathrm{n}=8]$ ), o de los OFZ producidos localmente (a $22,5 \%[n=8]$ y $10 \%[n=6]$ ).

Las formulaciones locales se prepararon en el Departamento de Farmacología de la Universidad de San Marcos, utilizando OFZ p.a (Spectrum Laboratory Products Inc., Gardena, CA, EUA) con los siguientes agentes tensoactivos: carboximetilcelulosa de sodio, ácido cítrico, propilparabeno, metilparabeno y glicerina. Tanto el metil como el propil parabeno se disolvieron en agua desionizada a $85^{\circ} \mathrm{C}$ por agitación; de manera similar, se preparó una solución que contenía carboximetilcelulosa de sodio y ácido cítrico con agua desionizada a temperatura ambiente, luego se añadió glicerina y se disolvió por agitación. En esta solución, se añadió el OFZ (según las formulaciones) en partes y se mezcló por agitación fuerte. Una vez que la solución de OFZ se homogeneizó completamente, se añadió la solución con metil y propil parabeno y se mezcló por agitación hasta la incorporación total. Esta emulsión pasó a través de un molino de rodillos para homogeneizar la mezcla, que se completó hasta alcanzar un peso final de $100 \mathrm{~kg}$ mediante la adición de agua desionizada. Todos los procedimientos de fabricación y almacenamiento se realizaron bajo protección de la luz di- 
recta. Se utilizaron los productos en un periodo máximo de un mes después de su fabricación.

Se realizó la administración oral de OFZ (según el peso de los cerdos) con una jeringa dosificadora, bajo la supervisión de un veterinario. Las formulaciones se administraron en ayuno; se monitorearon los cerdos durante todo el experimento para observar cualquier efecto secundario, como anorexia y letargo, previamente descrito ${ }^{(12)}$. La eutanasia de los cerdos se realizó ocho semanas después del tratamiento con OFZ, usando una combinación de ketamina (10 mg/kg) más xilazina $(2 \mathrm{mg} / \mathrm{kg})$ por vía intramuscular y una sobredosis de pentobarbital sódico $(120 \mathrm{mg} / \mathrm{kg})$ por vía intravenosa. Se realizó una necropsia detallada en cada canal, y se extrajeron todos los quistes del músculo esquelético, corazón, lengua y el cerebro.

Los quistes se clasificaron como viables (vesículas con líquido transparente y un escólex claramente visible) o degenerados (vesículas blancas densas sin líquido ni escólex) y fueron resumidos utilizando la media geométrica (MG), según el tipo de muestra y tratamientos. Se compararon los porcentajes de eficacia en cada cerdo (número de quistes degenerados / número de quistes totales $\times 100$ ) y número de quistes (sanos y degenerados), según tratamientos mediante análisis de Kruskal Wallis, utilizando el paquete estadístico RStudio v1.0.143. El Comité de Ética Animal de la FMV-UNMSM aprobó los protocolos de estudio, de acuerdo con las pautas establecidas por la Oficina de los Laboratorios de Bienestar Animal (Institutos Nacionales de Salud [NIH], EUA).

\section{HALLAZGOS}

No se observaron reacciones adversas durante todo el periodo experimental. Dos cerdos, con los números de identificación 9148 (formulación comercial del OFZ) y 9264 (formulación local al 22,5\%), respectivamente, no presentaron quistes ni lesiones degeneradas en absoluto, y fueron excluidos de los análisis.

No se encontraron quistes viables; solo se encontraron quistes degenerados en las muestras de músculo esquelético, corazón y lengua (Tabla 1, Figura 1A y 1B), lo cual demuestra una eficacia cisticida del $100 \%$ para las tres formulaciones de OFZ. Asimismo, las cargas parasitarias con cisticercos degenerados no fueron diferentes entre los grupos $(p=0,837)$. Se encontraron quistes cerebrales viables y degenerados en los cerdos tratados con todas las formulaciones de OFZ (Tabla 1), siendo los porcentajes de eficacia del $65 \%$ para la formulación comercial, $47 \%$ y $31 \%$ para las formulaciones locales a $22,5 \%$ y $10 \%$, respectivamente ( $\mathrm{p}=0,355$, Figura $1 \mathrm{C}$ ). La carga parasitaria con cisticercos cerebrales viables fue menor en el grupo tratado con la formulación comercial de OFZ (MG: 2), en comparación con los tratados con las formulaciones de OFZ producidas localmente en 22,5\% (MG: 3,6) y 10\% (MG: 5,7), respectivamente, aunque estas diferencias no fueron estadísticamente significativas $(\mathrm{p}=0,185)$.

\section{DISCUSIÓN}

Nuestro estudio demostró una eficacia similar entre las dos formulaciones de OFZ locales y la comercial contra los quistes musculares, como se ha demostrado consistentemente en los estudios previos ${ }^{(6,7)}$. Independientemente de la formulación utilizada, todos los quistes musculares se eliminaron después de una dosis única de $30 \mathrm{mg} / \mathrm{kg}$, y ocho semanas después solo se observaron quistes degenerados en la musculatura de los cerdos ${ }^{(12)}$. Dos cerdos tratados con la formulación comercial y local de OFZ al $22,5 \%$ respectivamente, no tuvieron quistes ni lesiones en la necropsia. Es posible que estos cerdos hayan sido diagnosticados erróneamente con la prueba de lengua; pero también es posible que la eliminación de todos los rastros de infección sea resultado de infecciones leves, dado que el tiempo efectivo para matar los quistes en el cerdo depende principalmente de la intensidad de la infección ${ }^{(12,13)}$.

En comparación con otros benzimidazoles como albendazol, el OFZ se absorbe más fácilmente desde el tracto gastrointestinal, lo que se correlaciona con la alta concentración plasmática del fármaco que se observa en los cerdos a tan solo dos horas después del tratamiento ${ }^{\left({ }^{(9)}\right.}$. Esta rápida absorción del OFZ en los cerdos también se ve favorecida por su estructura monogástrica, en contraste con los rumiantes, donde una cantidad significativa del fármaco se retiene junto al alimento digerido en el rumen ${ }^{(14)}$. La sustitución aromática en el átomo de azufre de la estructura química del OFZ también proporciona un tiempo prolongado de exposición sistémica del fármaco al parásito, lo que puede explicar su mayor eficacia cisticida ${ }^{(9)}$. Aunque los procedimientos de fabricación de fármacos pueden influir en la farmacocinética de las formulaciones del OFZ, como se describió previamente ${ }^{(9)}$, una concentración plasmática alta después de una dosis de $30 \mathrm{mg} / \mathrm{kg}$ es suficiente para matar todos los cisticercos en la musculatura.

Se ha reportado previamente niveles bajos de eficacia de OFZ contra quistes cerebrales ${ }^{(15)}$; sin embargo, las razones aún no están del todo claras. Las tres formulaciones de OFZ probadas en nuestro estudio fueron altamente eficaces contra los quistes musculares, pero mostraron una menor eficacia contra las infecciones cerebrales (se encontraron quistes viables en el cerebro de los cerdos durante las necropsias). Se observó que el grupo tratado con OFZ comercial presentó menor cantidad de quistes viables en el cerebro que los grupos tratados con OFZ local. Sin embargo, no se puede excluir una verdadera diferencia en la eficacia contra los quistes cerebrales, ya que las diferencias no estadísticas entre los tratamientos pueden reflejar la falta de poder estadístico debido a un tamaño de muestra pequeño. En cualquier caso, la baja eficacia del OFZ contra los quistes cerebrales no es un inconveniente para sus aplicaciones en los programas de control de cisticercosis ${ }^{(16)}$. 
Tabla 1. Distribución detallada de quistes viables y degenerados en cerdos, y porcentajes de eficacia según las muestras de tejido y los grupos de tratamiento.

\begin{tabular}{|c|c|c|c|c|c|c|c|c|c|}
\hline \multirow[b]{2}{*}{ Grupo } & \multirow{2}{*}{$\begin{array}{l}\text { Número de } \\
\text { cerdo }\end{array}$} & \multicolumn{2}{|c|}{ Músculo esquelético } & \multicolumn{2}{|c|}{ Corazón } & \multicolumn{2}{|c|}{ Lengua } & \multicolumn{2}{|c|}{ Cerebro } \\
\hline & & $\begin{array}{l}\text { Quistes } \\
\text { viables }\end{array}$ & $\begin{array}{c}\text { Quistes } \\
\text { degenerados }\end{array}$ & $\begin{array}{l}\text { Quistes } \\
\text { viables }\end{array}$ & $\begin{array}{c}\text { Quistes } \\
\text { degenerados }\end{array}$ & $\begin{array}{l}\text { Quistes } \\
\text { viables }\end{array}$ & $\begin{array}{c}\text { Quistes } \\
\text { degenerados }\end{array}$ & $\begin{array}{l}\text { Quistes } \\
\text { viables }\end{array}$ & $\begin{array}{c}\text { Quistes } \\
\text { degenerados }\end{array}$ \\
\hline \multirow{9}{*}{1} & 302 & 0 & 390 & 0 & 16 & 0 & 17 & 0 & 1 \\
\hline & 312 & 0 & 8236 & 0 & 114 & 0 & 268 & 1 & 8 \\
\hline & 314 & 0 & 977 & 0 & 12 & 0 & 23 & 0 & 2 \\
\hline & 271 & 0 & 132 & 0 & 0 & 0 & 7 & 0 & 4 \\
\hline & 222 & 0 & 1130 & 0 & 0 & 0 & 43 & 1 & 0 \\
\hline & 301 & 0 & 38 & 0 & 0 & 0 & 9 & 8 & 0 \\
\hline & 7200 & 0 & 9281 & 0 & 75 & 0 & 70 & 0 & 0 \\
\hline & $\mathrm{MG}^{\mathrm{a}}$ & - & $773,1^{\text {a }}$ & - & $35,8^{\mathrm{a}}$ & - & $29,6^{\mathrm{a}}$ & $2,0^{\mathrm{a}}$ & $2,8^{\mathrm{a}}$ \\
\hline & Eficacia (\%) ${ }^{\mathrm{b}}$ & - & 100,0 & - & 100,0 & - & 100,0 & - & 64,8 \\
\hline \multirow{9}{*}{2} & 303 & 0 & 6943 & 0 & 113 & 0 & 115 & 2 & 0 \\
\hline & 308 & 0 & 2943 & 0 & 67 & 0 & 63 & 7 & 11 \\
\hline & 313 & 0 & 1187 & 0 & 32 & 0 & 34 & 44 & 6 \\
\hline & 349 & 0 & 173 & 0 & 19 & 0 & 11 & 0 & 0 \\
\hline & 230 & 0 & 244 & 0 & 7 & 0 & 23 & 1 & 0 \\
\hline & 9622 & 0 & 6724 & 0 & 4 & 0 & 78 & 0 & 0 \\
\hline & 9623 & 0 & 3456 & 0 & 0 & 0 & 36 & 1 & 4 \\
\hline & $\mathrm{MG}^{\mathrm{a}}$ & - & $1572,7^{\mathrm{a}}$ & - & $22,5^{\mathrm{a}}$ & - & $40,4^{\mathrm{a}}$ & $3,6^{\mathrm{a}}$ & $6,4^{\text {a }}$ \\
\hline & Eficacia (\%) ${ }^{b}$ & - & 100,0 & - & 100,0 & - & 100,0 & - & 47,3 \\
\hline \multirow{8}{*}{3} & 304 & 0 & 1128 & 0 & 17 & 0 & 26 & 0 & 1 \\
\hline & 306 & 0 & 5014 & 0 & 13 & 0 & 50 & 4 & 11 \\
\hline & 309 & 0 & 516 & 0 & 9 & 0 & 110 & 43 & 2 \\
\hline & 0032 & 0 & 4088 & 0 & 38 & 0 & 67 & 2 & 0 \\
\hline & 7185 & 0 & 5376 & 0 & 203 & 0 & 167 & 6 & 26 \\
\hline & 7186 & 0 & 380 & 0 & 0 & 0 & 10 & 3 & 1 \\
\hline & $\mathrm{MG}^{\mathrm{a}}$ & - & $1702,7^{\mathrm{a}}$ & - & $27,4^{\mathrm{a}}$ & - & $50,2^{\mathrm{a}}$ & $5,7^{\mathrm{a}}$ & $3,6^{\mathrm{a}}$ \\
\hline & Eficacia (\%) ${ }^{\mathrm{b}}$ & - & 100,0 & - & 100,0 & - & 100,0 & - & 30,6 \\
\hline
\end{tabular}

Eficacia: Proporción de cisticercos degenerados sobre cisticercos totales (sanos + degenerados)

1: Formulación comercial de oxfendazol (Synanthic $\left.{ }^{\circ} 9,06 \%\right) ; 2$ : Formulación local de oxfendazol (22,5\%); 3: Formulación local de oxfendazol (10\%)

${ }^{\text {a }}$ Media geométrica (MG), ${ }^{\mathrm{b}}$ promedio

Nuestro estudio tiene algunas limitaciones a considerar. Debido a la poca cantidad de quistes cerebrales viables, no evaluamos el efecto de las formulaciones locales sobre la viabilidad del quiste. Además, no pudimos diferenciar los quistes degenerados debido al tratamiento o a la evolución natural de la parasitosis. Si bien no pudimos estimar la carga parasitaria inicial en los cerdos, el análisis del número de cisticercos degenerados en la necropsia no demostró diferencias significativas entre los grupos, lo que sugiere que las cargas parasitarias eran similares. Nuestros resultados fueron obtenidos en condiciones controladas libres de infección, lo que puede limitar nuestra capacidad de establecer si las formulaciones locales son tan eficaces como las formulaciones comerciales en condiciones naturales donde los cerdos se infectan repetidamente. Un estudio previo mostró que los cerdos con cisticercosis tratados con una formulación comercial de OFZ estaban protegidos de nuevas infecciones durante al menos tres meses ${ }^{(17)}$.

La protección que surge después del tratamiento con OFZ parece ser consecuencia de la activación del sistema inmune contra los antígenos del cisticerco en degeneración ${ }^{(17,18)}$ y aparentemente es independiente de la formulación utilizada. Además, es necesario evaluar si las formulaciones locales de OFZ a $30 \mathrm{mg} / \mathrm{kg}$ también son eficaces contra nematodos en cerdos, como ha sido reportado con una formulación comercial previamente ${ }^{(19)}$.

En comparación con otros antiparasitarios que requieren dosis múltiples o presentan efectos secundarios ${ }^{(20)}$, el OFZ es seguro y altamente eficaz contra la cisticercosis porcina a una dosis de $30 \mathrm{mg} / \mathrm{kg}$. Las formulaciones locales de OFZ son fáciles de preparar y no requieren procedimientos sofisticados más allá del uso de agentes tensoactivos para mejorar su estabilidad. Desde un punto de vista práctico, las formulaciones locales de OFZ altamente concentradas que requieran menos volumen por dosis pueden proporcionar una alternativa práctica para dosificar fármacos en cerdos de áreas rurales y reducir el riesgo de neumonía por aspiración durante su administración. Nuestros resultados demuestran que las formulaciones locales de OFZ son altamente eficaces 


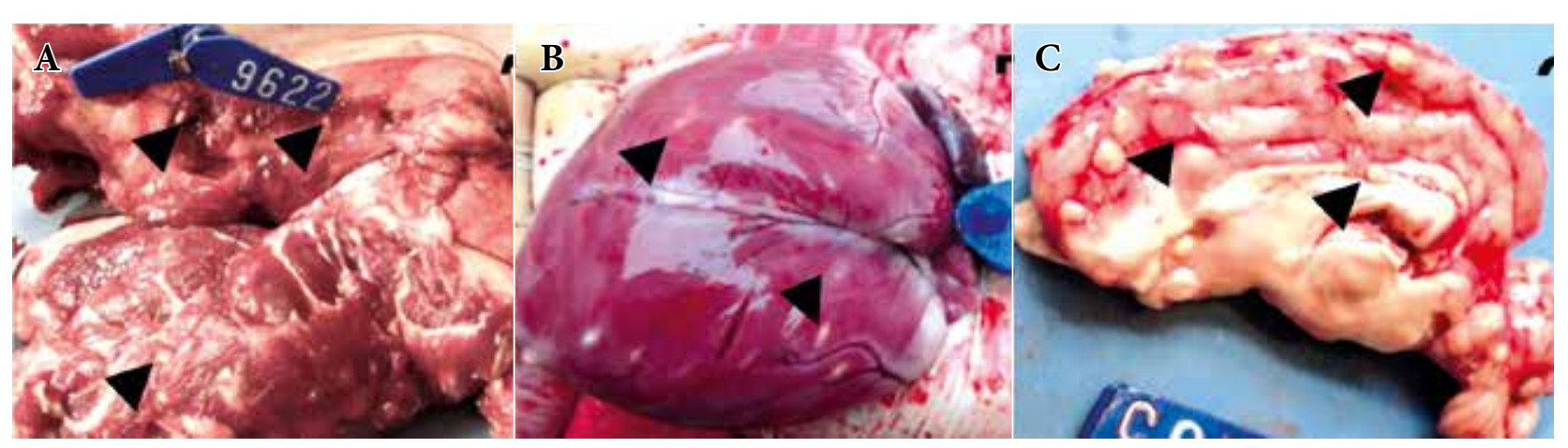

Figura 1. Vista macroscópica de los quistes en canales de cerdos ocho semanas después del tratamiento con oxfendazol. A) Cisticercos degenerados en músculo esquelético (flechas negras). B) Cisticercos degenerados en músculo cardiaco (flechas negras). C) Cisticercos degenerados en cerebro (flechas negras).

contra cisticercosis porcina y se requieren estudios adicionales para corroborar su eficacia en condiciones de campo.

Agradecimientos: Agradecemos al personal del Grupo de Trabajo de Cisticercosis en Perú por su colaboración durante el diseño y la implementación del experimento. Nuestro agradecimiento especial a Carlos Angulo, Elton Sánchez y Luis Villarreal, personal veterinario de la Universidad de San Marcos durante la fase experimental del estudio.

Otros miembros del Grupo de Trabajo en Cisticercosis en Perú: Manuela Verástegui, PhD; Mirko Zimic PhD (coordinación general); Isidro Gonzáles, MD; Herbert Saavedra, MD; Sofía Sánchez, MD; Manuel Martínez, MD (Instituto Nacional de Ciencias Neurológicas, Lima, Perú); Saúl Santivañez, MD, PhD; Holger Mayta, PhD; Mónica Pajuelo, PhD (Universidad Peruana Cayetano Heredia, Lima, Perú); Eloy Gonzales-Gustavson, DVM, PhD; César Gavidia, DVM, PhD (Universidad Nacional Mayor de San Marcos); Luz M. Moyano, MD; Ricardo Gamboa, MSc, Percy Vilchez, MSc; Claudio Muro (Programa de Eliminación de Cisticercosis, Tumbes, Perú);
Seth O'Neal, MD, MPH (Oregon Health and Sciences University, Portland, EUA); Sukwan Handali, MD; John Noh (Centers for Disease Control, Maryland, EUA); Theodore Nash, MD (National Institute of Allergy and Infectious Diseases, National Institutes of Health, Maryland, EUA); John Friedland, PhD (Imperial College, London, Reino Unido).

Contribuciones de los autores: HHG, RG y AEG concibieron el estudio; GA, JFC, LG y AVC participaron en el diseño experimental; GA y JAB, en la recolección de muestras y análisis de datos; GA y AVC, en la redacción del primer borrador del manuscrito; GA, ANV, LGP, HHG y AEG, en la redacción crítica del artículo y la versión final del manuscrito. Todos los autores han leído y aprobado la versión final.

Financiamiento: Este estudio fue financiado por el Centro Internacional Fogarty/NIH (becas de capacitación números D43TW008273-05 y D43TW001140).

Conflictos de intereses: Los autores declaran no presentar conflictos de intereses en la publicación del artículo.

\section{REFERENCIAS BIBLIOGRÁFICAS}

1. Garcia HH, Gonzalez AE, Gilman RH. Taenia solium Cysticercosis and Its Impact in Neurological Disease. Clin Microbiol Rev. 2020;33(3). doi: 10.1128/CMR.00085-19.

2. Gonzales I, Rivera JT, Garcia HH, Cysticercosis Working Group in P. Pathogenesis of Taenia solium taeniasis and cysticercosis. Parasite Immunol. 2016;38(3):136-46. doi: 10.1111/pim.12307.

3. Rajkotia Y, Lescano AG, Gilman RH, Cornejo C, Garcia HH, Cysticercosis Working Group of P. Economic burden of neurocysticercosis: results from Peru. Trans R Soc Trop Med Hyg. 2007;101(8):840-6. doi: 10.1016/j. trstmh.2007.03.008.

4. Lightowlers MW, Donadeu M. Designing a Minimal Intervention Strategy to Control Taenia solium. Trends Parasitol. 2017;33(6):426-34. doi: 10.1016/j.pt.2017.01.011.

5. Chalmers K. The efficacy of oxfendazole against natural infections of nematodes in cattle. N Z Vet J. 1978;26(6):162-4. doi: 10.1080/00480169.1978.34526.

6. Gonzales AE, Garcia HH, Gilman RH, Gavidia CM, Tsang VC, Bernal $\mathrm{T}$, et al. Effective, single-dose treatment or porcine cysticercosis with oxfendazole. Am J Trop Med Hyg. 1996;54(4):391-4.

7. Pondja A, Neves L, Mlangwa J, Afonso S, Fafetine J, Willingham AL 3rd, et al. Use of oxfendazole to control porcine cysticercosis in a high-endemic area of Mozambique. PLoS Negl Trop Dis. 2012;6(5): e1651. doi: 10.1371/ journal.pntd.0001651.
8. Megha GK, Aulakh RS, Singh BB. Effect of oxfendazole to control Taenia solium cysticercosis in pigs in Punjab state of India. J Parasit Dis. 2020;44(3):553-558. doi: 10.1007/s12639-020-01228-2.

9. Moreno L, Lopez-Urbina MT, Farias C, Domingue G, Donadeu M, Dungu B, et al. A high oxfendazole dose to control porcine cysticercosis: pharmacokinetics and tissue residue profiles. Food Chem Toxicol. 2012;50(10):3819-25. doi: 10.1016/j.fct.2012.07.023.

10. Chembensofu M, Mwape KE, Van Damme I, Hobbs E, Phiri IK, Masuku $\mathrm{M}$, et al. Re-visiting the detection of porcine cysticercosis based on full carcass dissections of naturally Taenia solium infected pigs. Parasit Vectors. 2017; 10:572. doi: https://doi.org/10.1186/s13071-017-2520-y.

11. Garcia HH, Gilman RH, Gonzalez AE, Verastegui M, Rodriguez S, Gavidia C, et al. Hyperendemic human and porcine Taenia solium infection in Peru. Am J Trop Med Hyg. 2003;68(3):268-75. doi: https://doi. org/10.4269/ajtmh.2003.68.268.

12. Iburg TM, Karlsson M, Spang F, Sikasunge CS, Johansen MV. The effect of oxfendazole treatment on muscle pathology in pigs infected with Taenia solium cysticercosis. Vet Parasitol. 2012;190(3-4):442-6. doi: 10.1016/j. vetpar.2012.07.007.

13. Sikasunge CS, Johansen MV, Willingham 3rd AL, Leifsson PS, Phiri IK. Taenia solium porcine cysticercosis: viability of cysticerci and persistency of antibodies and cysticercal antigens after treatment with oxfendazole. Vet Parasitol. 2008;158(1-2):57-66. doi: 10.1016/j.vetpar.2008.08.014. 
14. Steel JW, Hennessy DR. Influence of ruminal bypass on the pharmacokinetics and efficacy of benzimidazole anthelmintics in sheep. Int J Parasitol. 1999;29(2):305-14. doi: 10.1016/s0020-7519(98)00156-8.

15. Gonzalez AE, Falcon N, Gavidia C, Garcia HH, Tsang VC, Bernal T, et al. Time-response curve of oxfendazole in the treatment of swine cysticercosis. Am J Trop Med Hyg. 1998;59(5):832-6. doi: 10.4269/ ajtmh.1998.59.832.

16. Gilman RH, Gonzalez AE, Llanos-Zavalaga F, Tsang VC, Garcia HH, Cysticercosis Working Group in P. Prevention and control of Taenia solium taeniasis/cysticercosis in Peru. Pathog Glob Health. 2012;106(5):312-8. doi: 10.1179/2047773212Y.0000000045.

17. Gonzalez AE, Gavidia C, Falcon N, Bernal T, Verastegui M, Garcia $\mathrm{HH}$, et al. Protection of pigs with cysticercosis from further infections after treatment with oxfendazole. Am J Trop Med Hyg. 2001;65(1):158. doi: 10.4269/ajtmh.2001.65.15.

18. Liu YJ, Li QZ, Hao YH. Morphological changes to early stage Taenia solium cysticerci following oxfendazole treatment. Vet J. 2003;165(1):73-7. doi: 10.1016/s1090-0233(02)00124-7.

19. Alvarez L, Saumell C, Fuse L, Moreno L, Ceballos L, Domingue G, et al. Efficacy of a single high oxfendazole dose against gastrointestinal nematodes in naturally infected pigs. Vet Parasitol. 2013;194(1):70-4. doi: 10.1016/j.vetpar.2013.01.003.

20. Gonzalez AE, Garcia HH, Gilman RH, Lopez MT, Gavidia C, McDonald J, et al. Treatment of porcine cysticercosis with albendazole. Am J Trop Med Hyg. 1995;53(5):571-4. doi: 10.4269/ajtmh.1995.53.571. 